\title{
A Small Gain Framework for Networked Cooperative Teleoperation *
}

\author{
Ilia G. Polushin* Sergey N. Dashkovskiy** \\ * University of Western Ontario, London, ON, N6A 5B9, Canada \\ (e-mail: ipolushin@eng.uwo.ca). \\ ** Universität Bremen, Postfach 330440, 28334 Bremen, Germany \\ (e-mail:dsn@math.uni-bremen.de)
}

\begin{abstract}
A small gain approach for stability analysis of cooperative network-based force reflecting teleoperator systems is presented. The approach is based on a new version of inputto-output stability (IOS) nonlinear small gain theorem that is applicable to stability analysis of large-scale network-based interconnections. Using the small-gain theorem presented, we show that stability of the cooperative teleoperator system can always be achieved by choosing some of the system' gains appropriately.
\end{abstract}

Keywords: Nonlinear systems, Teleoperation, Stability analysis, Communication networks, Large-scale systems

\section{INTRODUCTION}

Teleoperation over communication networks has recently attracted significant attention due to its high flexibility, accessibility, and relatively low cost (Goldberg and Siegwart (2002)). The primary purpose of teleoperator systems is to make it possible for a human operator to execute a manipulation task remotely. A typical networked teleoperator system consists of two (or more) manipulators, called master(s) and slave(s), that are connected through a communication network. The master manipulator is manually controlled by the human operator, while the slave executes the task by following the motion of the master. In order to let the human operator feel the interaction with the task, the haptic data (slave positions/velocities as well as the interaction forces between the slave and the environment) may be transmitted back to the master site and displayed to the human operator through some sort of haptic interface. In cooperative teleoperator systems, multiple teleoperators perform tasks on the same environment (Sirouspour (2005); Wang et al. (2003)). Cooperative teleoperation enables collaboration between human operators that are geographically separated, and may lead to drastic improvement in handling capabilities, dexterity, as well as task completion time. Typical examples of applications include different assembly tasks, handling of toxic/radioactive materials and collaborative telesurgery. A structure of cooperative network-based teleoperator system is shown in figure 1. Specific features that makes such a system difficult for stability analysis include multiple communication constraints imposed by networks between the masters and the corresponding slaves, as well as possibility of slave-slave interactions through the common envi-

\footnotetext{
* The work of I. G. Polushin is partially supported by the Natural Sciences and Engineering Research Council of Canada. The work of S. N. Dashkovskiy is supported by the German Research Foundation (DFG) as part of the Collaborative Research Center 637 "Autonomous Cooperating Logistic Processes".
}

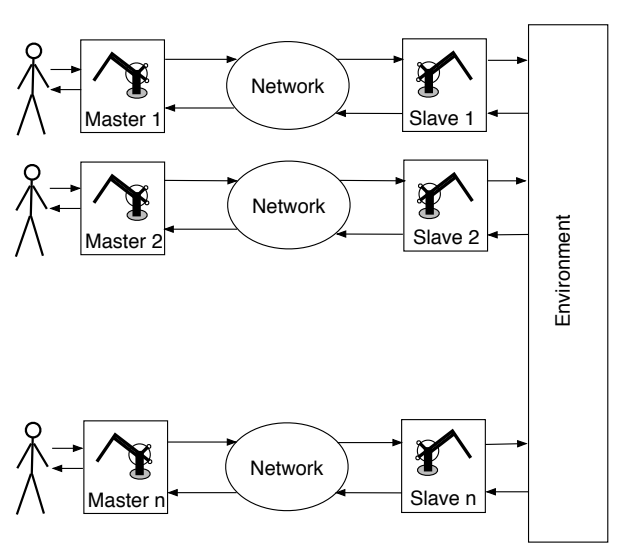

Fig. 1. Cooperative network-based teleoperator system

ronment. In particular, popular passivity/scatering/wave variable based approaches (Anderson and Spong (1989); Niemeyer and Slotine (2004)) are generally not applicable to cooperative teleoperation due to nonpassive slave-slave interactions.

One possible alternative to the passivity-based approaches is a design based on the small-gain arguments. Small-gain theorems are among the most powerful tools in analysis and control of interconnected nonlinear systems (Jiang (2004)). Direct application of the small-gain arguments to stability of cooperative network-based teleoperator systems, however, encounters significant difficulties. First, a cooperative teleoperator system consists of multiple master-slave pairs that interact through environment, which generally results in an interconnection structure more complex than a simple feedback interconnection. Second, communication over networks imposes communication constraints that include time-varying discontinuous possibly unbounded communication delays and possible packet losses. Also, in teleoperator systems, stability with required gain usually cannot be achieved globally, which 
implies that an appropriate version of the small-gain theorem must admit stability properties of subsystems to be satisfied within a compact subset of state space and a compact range of inputs rather than globally (which corresponds to stability with finite restrictions as well as bounded domain of small-gain conditions). Although a number of nonlinear small-gain theorems presented in the literature addressed some of the above described issues (see, for example, (Dashkovskiy et al. (2007); Karafyllis and Jiang (2009); Tiwari et al. (2009); Dashkovskiy et al. (2010)) for small gain conditions for interconnection of multiple subsystems, (Tiwari et al. (2009)) for the case of bounded communication delays, (Polushin et al. (2009)) for the case of discontinuous time-varying unbounded communication delays, and (Teel (1996); Polushin et al. (2009)) for the case of finite restrictions), no small-gain results exist that would directly fit the specific restrictions of the networked cooperative bilateral teleoperator systems.

In this paper, we present a small-gain framework for stability analysis of cooperative network-based force reflecting teleoperator systems. First, we formulate and prove a new version of input-to-output stability (IOS) small gain theorem that is applicable to stability analysis of large-scale network-based interconnections where the subsystems are assumed to be IOS with finite restrictions. As an application of this result, we consider a cooperative network-based teleoperator system with a typical interconnection structure. Using the small-gain theorem presented, we show that stability of the cooperative teleoperator system can always be achieved by choosing some of the system' gains appropriately. The structure of the paper is as follows. In Section 2, we formulate and prove a new version of the IOS small gain theorem. In Section 3, the IOS small-gain theorem is applied to stability analysis of the networkbased cooperative teleoperator system.

\subsection{Preliminaries}

Throughout the paper, the following standard notations are used. Let $\mathbb{R}_{+}$be the set of nonnegative real numbers, $\mathbb{R}_{+}:=[0,+\infty)$. A continuous function $\gamma: \mathbb{R}_{+} \rightarrow \mathbb{R}_{+}$is said to belong to class $\mathcal{G}(\gamma \in \mathcal{G})$ if it is strictly increasing; a function $\gamma \in \mathcal{G}$ belongs to class $\mathcal{K}(\gamma \in \mathcal{K})$ if it satisfies $\gamma(0)=0$; also, a function $\gamma \in \mathcal{K}$ belongs to class $\mathcal{K}_{\infty}$ if $\gamma(s) \rightarrow \infty$ as $s \rightarrow \infty$.

When analyzing stability of cooperative teleoperator systems, one deals with multiple inputs - multiple outputs (MIMO) systems where each input-output pair has a specific gain function associated with it. For simplicity of notations in MIMO case, it is convenient to use multivariable extensions of the classes $\mathcal{G}, \mathcal{K}, \mathcal{K}_{\infty}$, defined as follows. Let $\mathbb{R}_{+}^{n}$ be the positive orthant in $\mathbb{R}^{n}$, i.e., $\mathbb{R}_{+}^{n}:=\left\{x \in \mathbb{R}^{n}, x_{i} \geq 0\right.$ for all $\left.i=1, \ldots, n\right\}$. Given a set $\Gamma_{i j}: \mathbb{R}_{+} \rightarrow \mathbb{R}_{+}, i \in\{1, \ldots, n\}, j \in\{1, \ldots, m\}$, consider an associated map $\Gamma: \mathbb{R}_{+}^{m} \rightarrow \mathbb{R}_{+}^{n}$ defined according to the formula $\Gamma(s)=\left[(\Gamma(s))_{1}, \ldots,(\Gamma(s))_{n}\right]$, where

$$
(\Gamma(s))_{i}:=\max _{j \in\{1, \ldots, m\}} \Gamma_{i j}\left(s_{j}\right) .
$$

A map $\Gamma: \mathbb{R}_{+}^{m} \rightarrow \mathbb{R}_{+}^{n}$ is said to belong to class $\mathcal{G}^{n \times m}$ if and only if it can be associated with a set $\left\{\Gamma_{i j}\right\}, i \in\{1, \ldots, n\}$, $j \in\{1, \ldots, m\}$, where all $\Gamma_{i j} \in \mathcal{G}$. Classes $\mathcal{K}^{n \times m}$ and $\mathcal{K}_{\infty}^{n \times m}$ are defined analogously.
Some other notations used in the paper are as follows. Given two maps $\Gamma_{1}, \Gamma_{2}$ of appropriate dimensions, their composition is denoted by $\Gamma_{1} \circ \Gamma_{2}$ (i.e., $\Gamma_{1} \circ \Gamma_{2}(s):=$ $\Gamma_{1}\left(\Gamma_{2}(s)\right)$. Given a map $\Gamma \in \mathcal{G}^{n \times n}$ and a number $i \in$ $\mathbb{N}:=\{0,1, \ldots\}$, denote

$$
\Gamma^{i}:=\underbrace{\Gamma \circ \Gamma \circ \ldots \circ \Gamma}_{i \text { times }} .
$$

In particular, $\Gamma^{i}$ for $i=0$ is the identity map, $\Gamma^{0}(s):=s$. Further, given $x, y \in \mathbb{R}_{+}^{n}$, we write $x \geq y$ iff $x_{i} \geq y_{i}$ for all $i \in\{1, \ldots, n\}$, and $x \geq y$ otherwise. Relations $>$ and $\ngtr$ are defined analogously. The maximum of two or more vectors is calculated componentwise. For a finite set $X$, the number of its elements is denoted by $\# X$. Given $I \subset\{1, \ldots, n\}, I \neq \emptyset$, and $y \in \mathbb{R}_{+}^{n}$, denote $y_{I}:=\left\{y_{i}\right\}_{i \in I}$. Thus, $y_{I} \in \mathbb{R}^{\# I}$ is the projection of $y \in \mathbb{R}^{n}$ onto the subspace of $\mathbb{R}^{n}$ spanned by the basis vectors $\left\{e_{i}\right\}, i \in I$. The projection operator $y \rightarrow y_{I}$ is denoted by $\mathbb{P}_{I}$; thus, $y_{I}:=\mathbb{P}_{I}(y)$.

\section{SMALL GAIN THEOREM FOR NETWORK-BASED INTERCONNECTIONS}

Below, we use the following notation borrowed from (Teel (1998)). Given functions $f: \mathbb{R} \rightarrow \mathbb{R}^{n}, t_{d}: \mathbb{R} \rightarrow \mathbb{R}_{+}$, by $f_{d}(t)$ we denote the restriction of $f$ on the interval $\left[t-t_{d}(t) ; t\right]$, i.e., $f_{d}(t)=\left\{f(s), s \in\left[t-t_{d}(t) ; t\right]\right.$. Consider a system described by functional differential equations (FDEs) of the form

$$
\begin{aligned}
\dot{x} & =f\left(x_{d}, u_{1 d}, \ldots, u_{m d}, w_{1 d}, \ldots, w_{q d}\right), \\
y_{1} & =g_{1}\left(x_{d}, u_{1 d}, \ldots, u_{m d}, w_{1 d}, \ldots, w_{q d}\right), \\
\vdots & \vdots \\
y_{p} & =g_{p}\left(x_{d}, u_{1 d}, \ldots, u_{m d}, w_{1 d}, \ldots, w_{q d}\right) .
\end{aligned}
$$

Here, $x_{d}$ is a state, $x \in \mathbb{R}^{n}, u_{1}, \ldots u_{m}$ are finitedimensional control inputs, $y_{1}, \ldots y_{p}$ are finite-dimensional outputs, and $w_{1}, \ldots w_{q}$ are finite-dimensional disturbance inputs. According to the notation introduced above, the right-hand side of (1) depends on state and input trajectories restricted on the interval $\left[t-t_{d}(t) ; t\right]$ for some $t_{d}: \mathbb{R} \rightarrow \mathbb{R}_{+}$. For regularity purposes, it is assumed that $f, g_{1}, \ldots, g_{p}$ are Lipschitz continuous operators; also, $t_{d}(t)$ does not grow faster than time $t$; more precisely, the inequality $t_{d}\left(t_{2}\right)-t_{d}\left(t_{1}\right) \leq t_{2}-t_{1}$ holds for all $t_{1}, t_{2} \in \mathbb{R}$, $t_{2} \geq t_{1}$; also, $t-t_{d}(t) \rightarrow+\infty$ as $t \rightarrow+\infty$. Denote $|y|:=\left(\left|y_{1}\right|, \ldots,\left|y_{p}\right|\right)^{T} \in \mathbb{R}_{+}^{p} ; \mathbf{|} u \mathbf{I} \in \mathbb{R}_{+}^{m}, \quad \mathbf{I} w \mathbf{|} \in \mathbb{R}_{+}^{q}$ are defined analogously. It is assumed that system (1) satisfies the following local version of the input-to-output stability property (Sontag (2006)).

Assumption 1. The system (1) is input-to-output stable (IOS) with restrictions $\Delta_{x} \in \mathbb{R}_{+}, \Delta_{u} \in \mathbb{R}_{+}^{m}, \Delta_{w} \in \mathbb{R}_{+}^{q}$, i.e., there exist $\beta \in \mathcal{K}_{\infty}^{p \times 1}, \Gamma_{u} \in \mathcal{G}^{p \times m}, \Gamma_{w} \in \mathcal{G}^{p \times q}$, such that the conditions $\left|x_{d}(0)\right| \leq \Delta_{x}$, $\sup _{t \geq 0}\left|u_{d}(t)\right| \leq \Delta_{u}$, $\sup _{t>0}\left|w_{d}(t)\right| \leq \Delta_{w}$ imply that the solutions of (1) are well defined for $t \in[0,+\infty)$, and the following inequalities hold

$$
\sup _{t \geq 0}|y(t)| \leq \max \left\{\begin{array}{c}
\beta\left(\left|x_{d}(0)\right|\right), \\
\Gamma_{u}\left(\sup _{t \geq 0}\left|u_{d}(t)\right|\right), \\
\Gamma_{w}\left(\sup _{t \geq 0}\left|w_{d}(t)\right|\right)
\end{array}\right\},
$$


$\limsup _{t \rightarrow+\infty}|y(t)| \leq \max \left\{\begin{array}{l}\Gamma_{u}\left(\limsup _{t \rightarrow+\infty}\left|u_{d}(t)\right|\right) \\ \Gamma_{w}\left(\limsup _{t \rightarrow+\infty}\left|w_{d}(t)\right|\right)\end{array}\right\}$

In this work, we address the situation where different input and output channels of the system (1) are pairwise interconnected through communication network. The existence of a communication network may impose significant communication constraints such as time-varying discontinuous possibly unbounded communication delays as well as perturbations due to transmission errors, information losses and quantization. The communication constraints imposed on $j$-th input, where $j \in\{1, \ldots, m\}$, are described according to the formula

$$
\left|u_{j}(t)\right| \leq \max _{i \in\{1, \ldots, p\}}\left\{\mathcal{M}_{i}^{j}\left(\left|y_{i}^{*}\left(t-\tau_{i}^{j}(t)\right)\right|\right)\right\},
$$

where $y_{i}^{*}(t)=y_{i}(t)$ for $t \geq 0$ and $y_{i}^{*}(t)=0$ for $t<0$ (which implies that the connection is initiated at $t=0$ ), $\tau_{i}^{j}(t)$ is the communication delay induced by the network between $i$-th output and $j$-th input, and $\mathcal{M}_{i}^{j} \in \mathcal{G}$ is the corresponding "gain" function which, in particular, gives a room for possible amplification and/or distortion of the signal transmitted; it also provides an upper bound for errors due to quantization and information losses. All the communication delays satisfy the following assumption.

Assumption 2. The communication delays $\tau_{i}^{j}: \mathbb{R}_{+} \rightarrow \mathbb{R}_{+}$, $i \in\{1, \ldots, p\}, j \in\{1, \ldots, m\}$ are Lebesgue measurable functions with the following properties:

i) there exists a piecewise continuous function $\tau^{*}: \mathbb{R}_{+} \rightarrow$ $\mathbb{R}_{+}$satisfying $\tau^{*}\left(t_{2}\right)-\tau^{*}\left(t_{1}\right) \leq t_{2}-t_{1}$, such that the following inequalities hold for all $t \geq 0$

$$
\max _{\substack{i \in\{1, \ldots, p\} \\ j \in\{1, \ldots, m\}}} \tau_{i}^{j}(t) \leq \tau^{*}(t)
$$

ii)

$$
t-\max _{\substack{i \in\{1, \ldots, p\} \\ j \in\{1, \ldots, m\}}} \tau_{i}^{j}(t) \rightarrow+\infty \quad \text { as } \quad t \rightarrow+\infty . \bullet
$$

Assumption 2 is a relaxed version of similar assumptions used in Polushin et al. (2009). As shown in Polushin et al. (2009), it does not impose any restrictions on characteristics of the communication channel, and can always be satisfied in real-life networks unless the communication is totally lost on a semi-infinite time interval.

Now, let us denote $\Gamma:=\Gamma_{u} \circ \mathcal{M} \in \mathcal{G}^{p \times p}$, where

$$
\mathcal{M}:=\left[\begin{array}{ccc}
\mathcal{M}_{1}^{1} & \ldots & \mathcal{M}_{p}^{1} \\
\vdots & \ddots & \vdots \\
\mathcal{M}_{1}^{m} & \ldots & \mathcal{M}_{p}^{m}
\end{array}\right] \in \mathcal{G}^{m \times p}
$$

The following theorem gives small-gain conditions for IOS of system (1), (4).

Theorem 1. Consider an interconnected system (1), (4). Suppose Assumptions 1, 2 hold. Suppose also there exist $\delta, \Delta \in \mathbb{R}_{+}^{p}$, satisfying $\Gamma^{i-1}(\delta)<\Delta$ for all $i \in\{1, \ldots, p\}$, and $\mathcal{M}(\Delta) \leq \Delta_{u}$, such that the following conditions hold

$$
\Gamma(s) \nsupseteq s \quad \text { for all } s \in \mathbb{R}_{+}^{p}, s \leq \Delta, s \nless \delta .
$$

Then the interconnection (1), (4) is IOS with restrictions $\Delta_{x}^{*}, \Delta_{w}^{*}$, where

$$
\begin{aligned}
& \Delta_{x}^{*}:=\max \left\{\begin{array}{c}
s \in \mathbb{R}_{+}: s \leq \Delta_{x}, \\
\max _{i \in\{1, \ldots, p\}} \Gamma^{i-1} \circ \beta(s) \leq \Delta
\end{array}\right\}, \\
& \Delta_{w}^{*}:=\max \left\{\begin{array}{c}
s \in \mathbb{R}_{+}^{q}: s \leq \Delta_{w}, \\
\max _{i \in\{1, \ldots, p\}} \Gamma^{i-1} \circ \Gamma_{w}(s) \leq \Delta
\end{array}\right\} .
\end{aligned}
$$

More precisely, the conditions $\left|x_{d}(0)\right| \leq \Delta_{x}^{*}$, $\sup _{t \geq 0}\left|w_{d}(t)\right| \leq \Delta_{w}^{*}$ imply that the following inequalities

$$
\begin{aligned}
& \begin{array}{c}
\sup _{t \geq 0}|y(t)| \\
\leq \max _{i \in\{1, \ldots, p\}} \Gamma^{i-1}\left(\operatorname { m a x } \left\{\Gamma_{w}\left(\sup _{t \geq 0}\left|x_{d}(0)\right|\right),\right.\right. \\
\end{array} \\
& \limsup _{t \rightarrow+\infty}|y(t)| \\
& \leq \max _{i \in\{1, \ldots, p\}} \Gamma^{i-1}\left(\max \left\{\Gamma_{w}\left(\limsup _{t \rightarrow+\infty}\left|w_{d}(t)\right|\right), \delta\right\}\right) \text {. }
\end{aligned}
$$

hold along the trajectories of (1), (4).

\subsection{Proof of Theorem 1}

The proof makes use of the following two lemmas.

Lemma 2. Suppose $\Gamma \in \mathcal{G}^{p \times p}$ satisfies (7) for some $\delta, \Delta \in$ $\mathbb{R}_{+}^{p}$ such that $\Gamma^{i-1}(\delta)<\Delta$ for all $i \in\{1, \ldots, p\}$. Let $I \subset\{1, \ldots, p\}$ be an arbitrary nonempty index set, and $I^{c}:=\{1, \ldots, p\} \backslash I$. Then

$$
\Gamma_{I I}(s) \nsupseteq s \text { for all } s \in \mathbb{R}_{+}^{\# I}, s \leq \Delta_{I}, s \nless \delta_{I} .
$$

Proof. Pick an arbitrary $s_{0} \in \mathbb{R}_{+}^{\# I}$ such that $s_{0} \leq \Delta_{I}$ and $s_{0} \nless \delta_{I}$. Let $s \in \mathbb{R}_{+}^{p}$ be such that $s_{I}=s_{0}$ and $s_{I^{c}}=0$. Clearly, $s \leq \Delta$, and $s \nless \delta$. Condition (7) then implies that

$$
\left[\begin{array}{c}
s_{I} \\
0_{I^{c}}
\end{array}\right] \not \leq\left[\begin{array}{cc}
\Gamma_{I I} & \Gamma_{I I^{c}} \\
\Gamma_{I^{c} I} & \Gamma_{I^{c} I^{c}}
\end{array}\right]\left[\begin{array}{c}
s_{I} \\
0_{I^{c}}
\end{array}\right] \text {. }
$$

Clearly, $0 \leq \max \left\{\Gamma_{I^{c} I}\left(s_{I}\right), \Gamma_{I^{c} I^{c}}\left(0_{I^{c}}\right)\right\}$, therefore

$$
s_{I} \not \leq \max \left\{\Gamma_{I I}\left(s_{I}\right), \Gamma_{I^{c} I^{c}}\left(0_{I^{c}}\right)\right\} \geq \Gamma_{I I}\left(s_{I}\right) \text {. }
$$

The statement follows due to arbitrary choice of $s_{0}=s_{I}$. Lemma 3. Suppose (7) holds. Then for any $y \in \mathbb{R}_{+}^{p}$, $y \leq \Delta$, and any $v \in \mathbb{R}_{+}^{p}$ satisfying $\Gamma^{i-1}(v)<\Delta$ for all $i \in\{1, \ldots, p\}$, the condition

$$
y \leq \max \{\Gamma(y), v\}
$$

implies

$$
y \leq \phi:=\max _{i \in\{1, \ldots, p\}}\left\{\Gamma^{i-1}(\max \{v, \delta\})\right\} .
$$

Proof. Let $y \leq \Delta$. First, we claim that under the assumptions of Lemma 3,

$$
y \ngtr \max \{\delta, v\} .
$$

Indeed, assume the converse, i.e.,

$$
y>\max \{\delta, v\} \text {. }
$$

Taking into account (13), this implies $y \leq \Gamma(y)$. On the other hand, (16) also implies that $y \in(\delta, \bar{\Delta}]$, where $(\delta, \Delta]$ denotes the set of all vectors $y \in \mathbb{R}_{+}^{p}$ with $\delta<y \leq \Delta$, and therefore (7) implies $y \leq \leq \Gamma(y)$. This contradiction proves (15).

Now, inequality (15) implies that there exists a (possibly empty) index set $I_{1} \subset\{1, \ldots, p\}$ such that $I_{1}^{c}:=$ $\{1, \ldots, p\} \backslash I_{1} \neq \emptyset$, and $y_{I_{1}^{c}} \leq \max \left\{\delta_{I_{1}^{c}}, v_{I_{1}^{c}}\right\} \leq \phi_{I_{1}^{c}}$. If 
$I_{1}=\emptyset$, then (14) is proven. Otherwise, taking into account the last inequality, it follows from (13) that

$$
y_{I_{1}} \leq \max \left\{\Gamma_{I_{1} I_{1}}\left(y_{I_{1}}\right), \Gamma_{I_{1} I_{1}^{c}}\left(\delta_{I_{1}^{c}}\right), \Gamma_{I_{1} I_{1}^{c}}\left(v_{I_{1}^{c}}\right), v_{I_{1}}\right\} .
$$

Now, taking into account Lemma 2 , and using exactly the same line of reasoning as above, one can show that

$$
y_{I_{1}} \ngtr \max \left\{\delta_{I_{1}}, \Gamma_{I_{1} I_{1}^{c}}\left(\delta_{I_{1}^{c}}\right), \Gamma_{I_{1} I_{1}^{c}}\left(v_{I_{1}^{c}}\right), v_{I_{1}}\right\} .
$$

The last inequality precisely means that there exists a (possibly empty) index set $I_{2} \subset I_{1}, I_{2} \neq I_{1}$, such that $y_{I_{2}^{c}} \leq \phi_{I_{2}^{c}}$. Continuing this line of reasoning, after at most $p-1$ steps, we get (14). This completes the proof of Lemma 3.

Now, consider the system (1), (4). Suppose

$$
\left|x_{d}(0)\right| \leq \Delta_{x}^{*}, \quad \sup _{t \geq 0}\left|w_{d}(t)\right| \leq \Delta_{w}^{*} .
$$

Denote $\Delta^{*}:=\max \left\{\beta\left(\Delta_{x}^{*}\right), \Gamma_{w}\left(\Delta_{w}^{*}\right), \delta\right\}$. First, let us prove that

$$
\sup _{t \geq 0}|y(t)| \leq \Delta:=\max _{i \in\{1, \ldots, p\}} \Gamma^{i-1}\left(\Delta^{*}\right) .
$$

Note that, due to finite restriction $\Delta_{u}$ as well as bounded domain of the small-gain condition, the above inequality cannot be proven by applying small-gain arguments directly. Instead, let us consider the system (1) where the following interconnection constraints are imposed on each input $u_{j}, j \in\{1, \ldots, m\}$,

$$
\left|u_{j}(t)\right| \leq \epsilon \cdot \max _{i \in\{1, \ldots, p\}}\left\{\mathcal{M}_{i}^{j}\left(\left|y_{i}^{*}\left(t-\tau_{i}^{j}(t)\right)\right|\right)\right\},
$$

where $\epsilon \in[0,1]$. Using homotopy-like arguments similar to the ones used in Appendix of Teel (1996), we will show that (18) holds for all $\epsilon \in[0,1]$. First, for $\epsilon=0$, inequality (18) follows directly from Assumption 1. Fix an arbitrary $T \in(0,+\infty)$, and let $\nu \in \mathbb{R}_{+}^{p}, \nu>0$ be a vector with sufficiently small norm. Due to regularity (Lipschitz continuity) of the right-hand sides of system (1), the upper bound on trajectories of (1), (4) for $t \in[0, T]$ depends continuously on parameter $\epsilon$; more precisely, there exists $\epsilon^{*}>0$ such that the inequality

$$
\sup _{t \in[0, T]}|y(t)| \leq \Delta+\nu,
$$

holds as long as $\epsilon$ in (19) satisfies $\epsilon \in\left[0, \epsilon^{*}\right]$. Since $\epsilon^{*}<1$, for sufficiently small $\nu>0$ one has $\epsilon^{*} \cdot \mathcal{M}(\Delta+\nu) \leq$ $\mathcal{M}(\Delta) \leq \Delta_{u}$. Combining this with (19), (20), we see that

$$
\sup _{t \in\left[-t_{d}(0), T\right]}|u(t)| \leq \Delta_{u}
$$

i.e., the restriction on $u$ is met for all $t \in[0, T]$. Now, using (2), one gets

$$
\begin{gathered}
\sup _{t \in[0, T]}|y(t)| \\
\leq \max \left\{\Delta^{*}, \Gamma_{u} \circ\left(\epsilon^{*} \cdot \mathcal{M}\right)\left(\sup _{t \in[0, T]}|y(t)|\right)\right\},
\end{gathered}
$$

as long as $\epsilon$ in (19) satisfies $\epsilon \in\left[0, \epsilon^{*}\right]$. Furthermore, since $0<\epsilon^{*}<1$ and $\Gamma_{u}(\cdot)$ is strictly increasing, it is easy to see that, for sufficiently small $\nu>0$, condition (7) implies that $\Gamma_{u} \circ\left(\epsilon^{*} \cdot \mathcal{M}\right)(s) \nsupseteq s$ holds for all $s \in \mathbb{R}_{+}^{p}$, such that $s \leq \Delta+\nu$ and $s \nless \delta$. Applying Lemma 3, we see that

$$
\sup _{t \in[0, T]}|y(t)| \leq \Delta
$$

holds as long as $\epsilon \in\left[0, \epsilon^{*}\right]$. Now, let $\epsilon_{\max } \in(0,1]$ be the maximal number such that $(23)$ holds for all $\epsilon \in\left[0, \epsilon_{\max }\right]$.
We claim that $\epsilon_{\max }=1$. Indeed, assume the converse, i.e., $\epsilon_{\max }<1$. Then, for sufficiently small $\nu>0$, it follows by continuity of trajectories that there exists $\epsilon^{* *} \in\left(\epsilon_{\max }, 1\right)$ such that $(20)$ holds for all $\epsilon \in\left[0, \epsilon^{* *}\right]$. Using exactly the same line of reasoning as above, one can see that in this case (23) holds for all $\epsilon \in\left[0, \epsilon^{* *}\right]$, which contradicts the definition of $\epsilon_{\max }$. Thus, $\epsilon_{\max }=1$. Due to arbitrary choice of $T \in(0,+\infty)$, this implies (18); also, (21) holds for all $T>0$, which means that the restriction on $u$ is met. Thus, (2) holds along the trajectories of (1), (4).

Now, combining (2), (4), we get

$$
\sup _{t \geq 0}|y(t)| \leq \max \left\{\begin{array}{c}
\beta\left(\left|x_{d}(0)\right|\right), \Gamma\left(\sup _{t \geq 0}|y(t)|\right), \\
\Gamma_{w}\left(\sup _{t \geq 0}\left|w_{d}(t)\right|\right)
\end{array}\right\} .
$$

Taking into account (7), (18), and applying Lemma 3 , we see that (10) holds. To prove (11), note that due to Assumption 2, part ii), we have

$$
\limsup _{t \rightarrow+\infty}\left|y_{i}^{*}\left(t-\tau_{i}^{j}(t)\right)\right|=\limsup _{t \rightarrow+\infty}\left|y_{i}^{*}(t)\right|
$$

holds for each $i \in\{1, \ldots, p\}, j \in\{1, \ldots, m\}$. Taking into account (4) as well as definition of $y^{*}(\cdot)$, we see that

$$
\limsup _{t \rightarrow+\infty}\left|u_{d}(t)\right| \leq \mathcal{M}\left(\limsup _{t \rightarrow+\infty}|y(t)|\right) \text {. }
$$

Combining the above inequality with (3), taking into account small-gain condition (7) as well as (18), and applying Lemma 3, we get (11). The proof of Theorem 1 is now complete.

It is worth mentioning that, for sufficiently large $p$, small gain condition (7) may be hard to check. In this case, one can use sufficient conditions for (7) provided by Lemma 4 below. Let $I_{1}, \ldots, I_{k} \subset\{1, \ldots, q\}, k \in\{1, q\}$, be nonempty index sets such that $\left(I_{1}, \ldots, I_{k}\right)=(1, \ldots, q)$. Consider the following patrition of $\Gamma$,

$$
\Gamma=\left[\begin{array}{ccc}
\Gamma_{I_{1} I_{1}} & \ldots & \Gamma_{I_{1} I_{k}} \\
\vdots & \ddots & \vdots \\
\Gamma_{I_{k} I_{1}} & \ldots & \Gamma_{I_{k} I_{k}}
\end{array}\right]
$$

A function of the form

$$
\Gamma_{I_{k_{1}} I_{k_{2}}} \circ \ldots \circ \Gamma_{I_{k_{p}} I_{k_{1}}} \in \mathcal{G}^{\# I_{k_{1}} \times \# I_{k_{1}}}
$$

where $k_{1}, \ldots, k_{p} \in\{1, \ldots, k\}, k_{1} \neq \ldots \neq k_{p}$, is said to be a minimal cycle of (partitioned) matrix-function $\Gamma \in \mathcal{G}^{p \times p}$. The following lemma provides sufficient conditions for (7). Lemma 4. The small gain condition (7) holds if there exists a partition of $\Gamma \in \mathcal{G}^{p \times p}$ of the form (24) such that for each $k_{1} \in\{1, \ldots, k\}$, each minimal cycle of (24) satisfies

$\Gamma_{I_{k_{1}} I_{k_{2}}} \circ \ldots \circ \Gamma_{I_{k_{p}} I_{k_{1}}}(s)<s$ for all $s \in\left[\delta_{k_{1}}, \bar{\Delta}_{k_{1}}\right],(26)$ where $\delta_{k_{1}}:=\mathbb{P}_{I_{k_{1}}}(\delta), \bar{\Delta}_{k_{1}}:=\mathbb{P}_{I_{k_{1}}}(\bar{\Delta})$, and $\bar{\Delta}:=$ $\max _{i \in\{1, \ldots, p\}} \Gamma^{i-1}(\Delta)$.

Proof. Assume the converse, i.e., there exists $s \in \mathbb{R}_{+}^{p}, s \leq$ $\Delta, s \nless \delta$ such that $\Gamma(s) \geq s$. The last inequality can be rewritten as follows

$$
s_{I_{j}} \leq \max _{r \in\{1, \ldots, k\} \backslash j}\left\{\Gamma_{I_{j} I_{r}}\left(s_{I_{r}}\right)\right\}, \quad j \in\{1, \ldots, k\} .
$$

Substituting inequalities (27) into each other and using (26), we arrive at contradiction.

Theorem 1 and Lemma 4 are applicable to stability analysis of a very general class of large scale network-based 
interconnections where the communication between subsystems are subject to constraints typical for communication networks such as the Internet. Below, these results are utilized to derive conditions for stability of a cooperative networked force-reflecting teleoperator system.

\section{SMALL-GAIN ANALYSIS OF THE COOPERATIVE TELEOPERATION SYSTEM}

In this section, Theorem 1 and Lemma 4 are applied to stability analysis of a networked cooperative teleoperator system shown schematically in Figure 1. The cooperative teleoperator system under consideration consists of $2 N$ manipulators, $N$ masters and $N$ slaves. For each $i \in\{1, \ldots, N\}, i$-th master is connected with $i$-th slave over a networked communication channel. All the slave manipulators interact with the same environment. For each $i \in\{1, \ldots, N\}, i$-th master subsystem is described as follows

$$
\begin{aligned}
& \dot{x}_{m i}=f_{m i}\left(x_{m i}, u_{m i}^{\{h\}}, u_{m i}^{\{s\}}\right), \\
& y_{m i}=g_{m i}\left(x_{m i}, u_{m i}^{\{h\}}, u_{m i}^{\{s\}}\right),
\end{aligned}
$$

where $u_{m i}^{\{h\}}$ is the force/torque input applied by the human operator, and $u_{m i}^{\{s\}}$ is the force reflection signal that arrives from the remote slave. It is assumed that each master subsystem is IOS; the corresponding gain matrix $\Gamma_{m i}$ has a form $\Gamma_{m i}=\left[\gamma_{m i}^{\{h\}}(\cdot), \gamma_{m i}^{\{s\}}(\cdot)\right] \in \mathcal{G}^{1 \times 2}$. It is assumed that gain $\gamma_{m i}^{\{h\}}(\cdot)$ is given and generally cannot be changed, but $\gamma_{m i}^{\{s\}}>0$ can be assigned arbitrarily small. Also, for each $i \in\{1, \ldots, N\}, i$-th slave subsystem is described as follows,

$$
\begin{aligned}
\dot{x}_{s i} & =f_{s i}\left(x_{s i}, u_{s i}^{\{m\}}, u_{s i}^{\{e\}}\right), \\
y_{s i}^{\{e\}} & =g_{s i}^{\{1\}}\left(x_{s i}, u_{s i}^{\{m\}}, u_{s i}^{\{e\}}\right), \\
y_{s i}^{\{f\}} & =g_{s i}^{\{2\}}\left(x_{s i}, u_{s i}^{\{m\}}, u_{s i}^{\{e\}}\right) .
\end{aligned}
$$

In the above equations, $x_{s i}$ is state of $i$-th slave, and $u_{s i}^{\{m\}}, u_{s i}^{\{e\}}$ are input signals that come from the remote master and the environment, respectively. Output $y_{s i}^{\{e\}}$ is applied to the environment, and $y_{s i}^{\{f\}}$ represents a "force reflection" signal transmitted back to the corresponding master site. The exact choice of $y_{s i}^{\{e\}}$ depends on the model of the slave-environment interaction, while the choice of $y_{s i}^{\{f\}}$ is determined by the force reflection scheme used. Each slave subsystem is assumed to be IOS. To be more specific, let us assume that the environment is described in terms of its impedance model, so $y_{s i}^{\{e\}}$ is actually the slave state (position+velocity), the signal reflected back to the master site is the environmental force, and the admittance of each slave w.r.t. environmental force can be adjusted arbitrarily. These assumptions result in IOS gain matrix of the form

$$
\Gamma_{s i}=\left[\begin{array}{cc}
1 & \gamma_{s i}^{\{e\}} \\
0 & 1
\end{array}\right],
$$

where $\gamma_{s i}^{\{e\}}>0$ can be assigned arbitrarily small. Each master-slave pair is interconnected through network communication channel, and the communication in both direction is subject to network-induced communication constraints, described for each $i=1, \ldots, N$ as follows,

$$
\begin{aligned}
u_{m i}^{\{s\}}(t) & :=y_{s i}^{\{f\}}\left(t-\tau_{b i}(t)\right)+\sigma_{b i}(t), \\
u_{s i}^{\{m\}}(t) & :=y_{m i}\left(t-\tau_{f i}(t)\right)+\sigma_{f i}(t) .
\end{aligned}
$$

In the above formulas, $\tau_{f i}, \tau_{b i}: \mathbb{R}_{+} \rightarrow \mathbb{R}_{+}$are the communication delays in $i$-th forward and backward channels, respectively, and $\sigma_{f i}, \sigma_{b i}$ are the corresponding measurement/estimation/quantization/transmission errors. All $\tau_{f i}$, $\tau_{b i}, i=1, \ldots, N$, are assumed to satisfy Assumption 2, while all $\sigma_{f i}, \sigma_{b i}, i=1, \ldots, N$, are assumed to be Lebesgue measurable uniformly essentially bounded signals.

The environment is described by the following equations

$$
\begin{aligned}
\dot{x}_{e} & =f_{e}\left(x_{e}, u_{e 1}^{\{s\}}, \ldots, u_{e N}^{\{s\}}, f_{e x t}\right), \\
f_{e n v 1} & =g_{e 1}\left(x_{e}, u_{e 1}^{\{s\}}, \ldots, u_{e N}^{\{s\}}, f_{e x t}\right), \\
\vdots & \vdots \\
f_{e n v N} & =g_{e N}\left(x_{e}, u_{e 1}^{\{s\}}, \ldots, u_{e N}^{\{s\}}, f_{e x t}\right) .
\end{aligned}
$$

Here, $x_{e}$ is a state of the environment, $u_{e i}^{\{s\}}$ is the input signal that comes from $i$-th slave subsystem, and $f_{\text {ext }}$ is an external force input which is considered as a disturbance and assumed to be uniformly essentially bounded. The environment has $N+1$ inputs; for our purposes, it is convenient to decompose them into $N$ "control" inputs $u_{e 1}^{\{s\}}$, $\ldots, u_{e N}^{\{s\}}$, and one "external" input $f_{\text {ext }}$. The environment is assumed to be IOS, and the corresponding IOS gain matrices have a form

$$
\Gamma_{e s}=\left[\begin{array}{ccc}
\gamma_{11}^{e s} & \ldots & \gamma_{1 N}^{e s} \\
\vdots & \ddots & \vdots \\
\gamma_{N 1}^{e s} & \cdots & \gamma_{N N}^{e s}
\end{array}\right] \in \mathcal{G}^{N \times N}, \quad \Gamma_{e f}=\left[\begin{array}{c}
\gamma_{1 e x t} \\
\vdots \\
\gamma_{N e x t}
\end{array}\right] \in \mathcal{G}^{N \times 1}
$$

respectively. The elements of $\Gamma_{e s}$ depend on properties of the environment (stiffness, geometry, etc), and generally cannot be adjusted. Each $f_{\text {envi }}, i=1, \ldots, N$, represents the interaction force between the environment and $i$ th slave; it is applied to the corresponding slave input. More precisely, the interconnection between the slave manipulators and the environment is described as follows,

$$
u_{e i}^{\{s\}}=y_{s i}^{\{e\}}, \quad u_{s i}^{\{e\}}=f_{\text {envi }}, \quad i=1, \ldots, N .
$$

In order to analyze the stability of the cooperative teleoperator system described above, we first rewrite the equations of the masters, slaves and environment in the form (1), and consequently represent interconnections (28), (29) in terms of interconnection matrix $\mathcal{M}$. The cooperative teleoperator system without interconnections has $4 N$ outputs ( $i$. e., $p=4 N$ in (1)); for our purposes it is convenient to reorder them as follows: $y_{1}=y_{m 1}, \ldots, y_{N}=y_{m N}$, $y_{N+1}=y_{s 1}^{\{e\}}, \ldots, y_{2 N}=y_{s N}^{\{e\}}, y_{2 N+1}=y_{s 1}^{\{f\}}, \ldots$, $y_{3 N}=y_{s N}^{\{f\}}, y_{3 N+1}=f_{\text {env } 1}, \ldots, y_{4 N}=f_{\text {env } N}$. Next, the system has $4 N$ control inputs (i.e., $m=4 N$ in (1)), they are reordered as follows: $u_{1}=u_{m 1}^{\{s\}}, \ldots, u_{N}=u_{m N}^{\{s\}}$, $u_{N+1}=u_{s 1}^{\{m\}}, \ldots, u_{2 N}=u_{s N}^{\{m\}}, u_{2 N+1}=u_{s 1}^{\{e\}}, \ldots$, $u_{3 N}=u_{s N}^{\{e\}}, u_{3 N+1}=u_{e 1}^{\{s\}}, \ldots, u_{4 N}=u_{e N}^{\{s\}}$. Also, the system has $3 N+1$ "disturbance" inputs $(q=N+1$ in (1), these are $u_{m i}^{\{h\}}, i=1, \ldots, N$, and $f_{\text {ext }}$; their particular order is not essential for our purposes. Finally, since all the subsystems are described by ODEs, we have $t_{d}(t) \equiv 0$ in (1). Thus defined system is IOS; moreover, for the 
above choice of inputs and outputs, the corresponding gain matrix $\Gamma_{u} \in \mathcal{G}^{4 N \times 4 N}$ has a form

$$
\Gamma_{u}=\left[\begin{array}{cccc}
\Gamma_{m e} & \mathbb{O} & \mathbb{O} & \mathbb{O} \\
\mathbb{O} & \mathbb{I} & \Gamma_{s e} & \mathbb{O} \\
\mathbb{O} & \mathbb{O} & \mathbb{I} & \mathbb{O} \\
\mathbb{O} & \mathbb{O} & \mathbb{O} & \Gamma_{e s}
\end{array}\right],
$$

where $\Gamma_{m e}:=\operatorname{diag}\left\{\gamma_{m 1}^{\{s\}}, \ldots, \gamma_{m N}^{\{s\}}\right\}$,

$\Gamma_{s e}:=\operatorname{diag}\left\{\gamma_{s 1}^{\{e\}}, \ldots, \gamma_{s N}^{\{e\}}\right\}$, and $\mathbb{I}$ is the identity function of the corresponding dimension.

Now, consider interconnections (28), (29). Let $\sigma^{*} \in \mathbb{R}_{+}^{N}$, $\sigma^{*}>0$ be the uniform upper bound for all measurement/estimation/quantization/transmission errors in (28), i.e.,

$$
\sup _{t \in[0,+\infty)} \max \left\{\left|\sigma_{f i}(t)\right|,\left|\sigma_{b i}(t)\right|\right\} \leq \sigma_{i}^{*}, \quad i \in\{1, \ldots, N\} .
$$

Let $\mathbb{I}_{\sigma^{*}} \in \mathcal{G}^{N \times N}$ be defined as follows $\mathbb{I}_{\sigma^{*}}(s):=s+\sigma^{*}$. In this case, the interconnection matrix has a form

$$
\mathcal{M}=\left[\begin{array}{cccc}
\mathbb{O} & \mathbb{O} & \mathbb{I}_{\sigma^{*}} & \mathbb{O} \\
\mathbb{I}_{\sigma^{*}} & \mathbb{O} & \mathbb{O} & \mathbb{O} \\
\mathbb{O} & \mathbb{O} & \mathbb{O} & \mathbb{I} \\
\mathbb{O} & \mathbb{I} & \mathbb{O} & \mathbb{O}
\end{array}\right] .
$$

Applying Theorem 1, one can see that the following small gain condition

$$
\Gamma(s):=\Gamma_{u} \circ \mathcal{M}(s) \nsupseteq s \quad \forall s \in \mathbb{R}_{+}^{4 N}, s \leq \Delta, s \nless \delta,
$$

implies that the closed-loop cooperative teleoperator system is stable with restrictions $\Delta_{x}^{*}, \Delta_{w}^{*}$ defined by (8), (9). Now, applying Lemma 4, one can derive that (30) is satisfied if the following inequalities hold

$$
\begin{aligned}
& \Gamma_{m e} \circ \mathbb{I}_{\sigma^{*}} \circ \Gamma_{e s} \circ \mathbb{I}_{\sigma^{*}}(s)<s \\
& \forall s \in \mathbb{R}_{+}^{N}, s \in\left[\mathbb{P}_{1}(\delta), \mathbb{P}_{1}\left(\max _{i \in\{1, \ldots, p\}} \Gamma^{i-1}(\Delta)\right)\right] \text {, } \\
& \mathbb{I}_{\sigma^{*}} \circ \Gamma_{m e} \circ \mathbb{P}_{\sigma^{*}} \circ \Gamma_{e s}(s)<s \\
& \forall s \in \mathbb{R}_{+}^{N}, s \in\left[\mathbb{P}_{2}(\delta), \mathbb{P}_{2}\left(\max _{i \in\{1, \ldots, p\}} \Gamma^{i-1}(\Delta)\right)\right] \text {, } \\
& \begin{array}{c}
\Gamma_{s e} \circ \Gamma_{e s}(s)<s \\
\forall s \in \mathbb{R}_{+}^{N}, s \in\left[\mathbb{P}_{2}(\delta), \mathbb{P}_{2}\left(\max _{i \in\{1, \ldots, p\}} \Gamma^{i-1}(\Delta)\right)\right],
\end{array} \\
& \Gamma_{e s} \circ \mathbb{I}_{\sigma^{*}} \circ \Gamma_{m e} \circ \mathbb{I}_{\sigma^{*}}(s)<s \\
& \forall s \in \mathbb{R}_{+}^{N}, s \in\left[\mathbb{P}_{3}(\delta), \mathbb{P}_{3}\left(\max _{i \in\{1, \ldots, p\}} \Gamma^{i-1}(\Delta)\right)\right] \text {, } \\
& \begin{array}{c}
\Gamma_{e s} \circ \Gamma_{s e}(s)<s \\
\forall s \in \mathbb{R}_{+}^{N}, s \in\left[\mathbb{P}_{4}(\delta), \mathbb{P}_{4}\left(\max _{i \in\{1, \ldots, p\}} \Gamma^{i-1}(\Delta)\right)\right],
\end{array} \\
& \Gamma_{e s} \circ \mathbb{I}_{\sigma^{*}} \circ \Gamma_{m e} \circ \mathbb{I}_{\sigma^{*}}(s)<s \\
& \forall s \in \mathbb{R}_{+}^{N}, s \in\left[\mathbb{P}_{4}(\delta), \mathbb{P}_{4}\left(\max _{i \in\{1, \ldots, p\}} \Gamma^{i-1}(\Delta)\right)\right],
\end{aligned}
$$

where $\mathbb{P}_{i}: \mathbb{R}_{+}^{4 N} \rightarrow \mathbb{R}_{+}^{N}, i=1, \ldots, 4$ are canonical projections defined by the formulas $\mathbb{P}_{1}(\delta)=[\mathbb{I} \mathbb{O}(\mathbb{O} \mathbb{O}] \delta$, $\mathbb{P}_{2}(\delta)=[\mathbb{O} \mathbb{I} \mathbb{O} \mathbb{O}] \delta$, etc., where $\mathbb{I}, \mathbb{O} \in \mathbb{R}^{N \times N}$ are unit and zero matrices, respectively. It is easy to check that conditions (31)-(36) can be met for any $\delta \in \mathbb{R}_{+}^{4 N}$ satisfying

$$
\delta>\left[\begin{array}{c}
0 \\
\sigma^{*} \\
\Gamma_{e s}\left(\sigma^{*}\right) \\
\Gamma_{e s}\left(\sigma^{*}\right)
\end{array}\right]
$$

and any sufficiently large $\Delta \in \mathbb{R}_{+}^{4 N}$, by an appropriate choice of $\Gamma_{m e}=\operatorname{diag}\left\{\gamma_{m 1}^{\{s\}}, \ldots, \gamma_{m N}^{\{s\}}\right\}$, and $\Gamma_{s e}=$ $\operatorname{diag}\left\{\gamma_{s 1}^{\{e\}}, \ldots, \gamma_{s N}^{\{e\}}\right\}$. Thus, the closed-loop cooperative teleoperator system can be made stable with arbitrary large restrictions and an arbitrary "offset" $\delta$ satisfying (37), by an appropriate choice of $\Gamma_{m e}$, and $\Gamma_{s e}$.

\section{REFERENCES}

Anderson, R.J. and Spong, M.W. (1989). Bilateral control of teleoperators with time delay. IEEE Trans. Aut.Contr., AC-34(5), 494-501.

Dashkovskiy, S., Rüffer, B., and Wirth, F.R. (2007). An ISS small gain theorem for general networks. Mathematics of Control, Signals, and Systems, 19(2), 93-122.

Dashkovskiy, S., Rüffer, B., and Wirth, F.R. (2010). Small gain theorems for large scale systems and construction of ISS Lyapunov functions. SIAM Journal of Control and Optimization, 48(6), 4089-4118.

Goldberg, K. and Siegwart, R. (eds.) (2002). Beyond Webcams: An Introduction to Online Robots. MIT Press.

Jiang, Z.P. (2004). Control of interconnected nonlinear systems: a small gain viewpoint. In M. de Queiroz, M. Malisoff, and P. Wolenski (eds.), Optimal Control, Stabilization, and Nonsmooth Analysis, 183-195. Springer-Verlag, Heidelberg.

Karafyllis, I. and Jiang, Z.P. (2009). A vector small-gain theorem for general nonlinear systems. In 48th IEEE Conference on Decision and Control and 28th Chinese Control Conference. Shanghai, P. R. China.

Niemeyer, G. and Slotine, J.J.E. (2004). Telemanipulation with time delays. International Journal of Robotics Research, 23(9), 873-890.

Polushin, I.G., Marquez, H.J., Tayebi, A., and Liu, P.X. (2009). A multichannel IOS small gain theorem for systems with multiple time-varying communication delays. IEEE Transactions on Automatic Control, 54(2), 404409.

Sirouspour, S. (2005). Modeling and control of cooperative teleoperation systems. IEEE Transactions on Robotics, $21(6), 1220-1225$.

Sontag, E.D. (2006). Input-to-state stability: Basic concepts and results. In P. Nistri and G. Stefani (eds.), Nonlinear and Optimal Control Theory, 163-220. SpringerVerlag, Berlin.

Teel, A.R. (1996). A nonlinear small gain theorem for the analysis of control systems with saturation. IEEE Transactions on Automatic Control, AC-41(9), 12561270.

Teel, A.R. (1998). Connections between Razumikhin-type theorems and the ISS nonlinear small gain theorem. IEEE Transactions on Automatic Control, AC-43(7), 960-964.

Tiwari, S., Wang, Y., and Jiang, Z.P. (2009). A nonlinear small-gain theorem for large-scale time-delay systems. In 48th IEEE Conference on Decision and Control and 28th Chinese Control Conference. Shanghai, P. R. China.

Wang, X.G., Moallem, M., and Patel, R.V. (2003). An Internet-based distributed multiple-telerobot system. IEEE Transactions on Systems, Man, and Cybernetics, Part A: Systems and Humans, 33(5), 627-633. 